\title{
Cyclic Nucleotide Biochemistry
}

National Cancer Institute

\section{Source}

National Cancer Institute. Cyclic Nucleotide Biochemistry. NCI Thesaurus. Code C18726.

The branch of biochemistry concerned with the metabolism of and biological pathways involving cyclic nucleotides. 